\title{
Random Walks for Deformable Image Registration
}

\author{
Dana Cobzas and Abhishek Sen \\ Computing Science, University of Alberta, Canada
}

\begin{abstract}
We introduce a novel discrete optimization method for nonrigid image registration based on the random walker algorithm. We discretize the space of deformations and formulate registration using a Gaussian MRF where continuous labels correspond to the probability of a point having a certain discrete deformation. The interaction (regularization) term of the corresponding MRF energy is convex and image dependent, thus being able to accommodate different types of tissue elasticity. This formulation results in a fast algorithm that can easily accommodate a large number of displacement labels, has provable robustness to noise and a close to global solution. We experimentally demonstrate the validity of our formulation on synthetic and real medical data.
\end{abstract}

\section{Introduction}

Image registration is a fundamental problem in medical imaging, central for many clinically relevant applications like statistical studies on a population of patients, analysis of disease progress and multi-modality fusion for better diagnosis and treatment. The registration problem can be formalized as finding the optimal transformation that aligns a source with a target image, based on a similarity score. Depending on the type of transformation, registration methods can be classified into global (rigid, affine) and local (non-linear, non-rigid). Global registration methods involve few parameters to be optimized and are thus wellposed, being constrained in the parameter space. Non-rigid registration methods estimate a dense deformation field that defines, for every location, a vector that locally aligns the two images. This is an inherently ill-posed problem due to the high dimensionality of the parameter space and therefore relies on regularization.

Several ways of imposing regularization have been proposed in the literature [1]. The popular free form deformation model (FFD) [2] restricts the parameter space to a set of control points that define a smooth interpolation field for the rest of the image. Alternative methods explicitly add a regularization term (e.g. fluid, elastic) in the registration energy, that is either optimized together with the data matching term 34, or applied as a separate smoothing process (demon's [5]). One other way of imposing regularization is to restrict the space of deformations to a Sobolev space [6]. Some effort has been made to adapt the regularization of deformations to local image content 78 ]. This is particularly important considering that different tissue deform differently and parts of the image might contain an abnormality that does not match the atlas. 
Nonlinear registration is traditionally formulated in a continuous domain and the optimal transformation is recovered using gradient descent. This estimation is often slow and suboptimal due to non-convexity of the energy functional that is optimized. Recently, few works have formulated deformable registration as a discrete labeling problem 910]. The space of deformations is discretized and the registration energy is formulated as a Markov random field (MRF) optimization. If the interaction energy is submodular, a graph cut method guarantees a good quality solution [110]. For more complex interaction terms, Glocker et al. 9] proposed a linear programming method that uses the primal dual principle.

In this paper we proposed an alternative discrete formulation of the registration problem based on the random walker algorithm [12 13. Our formulation is equivalent to a Gaussian MRF with an interaction (regularization) term that is convex and image dependent, thus being able to accommodate different types of tissue elasticity. Following the formulation from [13, we incorporate data similarity terms as 'priors' for the displacement labels. Unlike the graph cuts [10] or the primal dual method 9] that would only guarantee a good quality solution for the discrete registration, the random walker method finds a unique global minimum. Probabilities of a particular displacement label are calculated by solving a combinatorial Laplace equation. The random walker formulation of the registration problem results in a fast algorithm that can easily accommodate a large number of displacement labels and has provable robustness to noise 12 .

\section{Methods}

\subsection{An Energy Formulation of Deformable Registration}

Let $I$ and $J$ respectively be the reference (target) and the floating (source) $d$ dimensional images $I, J: \Omega \rightarrow \mathbb{R}, \Omega \subset \mathbb{R}^{d}$. Image registration seek an optimal transformation $T: \Omega \rightarrow \Omega$ that aligns the two images based on a similarity measure. In deformable registration, $T$ is usually expressed in terms of a displacement field $u$ as $T=\mathrm{Id}+u$, with the identity operator Id. $u$ is found as the minimum of an energy functional:

$$
u^{*}=\operatorname{argmin}_{u} E_{D}(I, J \circ T)+\alpha E_{R}(u)
$$

where $E_{D}$ is a data term that measures the similarity between the two images and $E_{R}$ is the regularization energy term. Expanding the two energy terms, and denoting the similarity measure with $\Phi$ and an image-dependent (adaptable) regularization function with $\Psi$ we get:

$$
u^{*}=\operatorname{argmin}_{u} \int_{\mathbf{x} \in \Omega} \Phi(I(\mathbf{x}), J(\mathbf{x}+u(\mathbf{x}))) d \mathbf{x}+\alpha \int_{\mathbf{x} \in \Omega} \Psi(\nabla J(\mathbf{x}), \nabla u(\mathbf{x})) d \mathbf{x}
$$

\subsection{Discrete Formulation for the Random Walker Algorithm}

Regarding $I$ and $J$ as discrete representations for the target and source image, we next formulate registration as a discrete optimization. We consider a discrete 
set of labels $\mathcal{L}=\left\{u^{1}, u^{2}, \ldots, u^{K}\right\}$ corresponding to a quantized version of the deformation space $u^{i} \in \mathcal{D}=\left\{\mathbf{d}^{1}, \mathbf{d}^{2}, \ldots, \mathbf{d}^{K}\right\}$. The registration problem becomes a labeling problem that seeks to assign an optimal label for each image location. A common model for representing such problems are MRFs. The pixel locations of the image are mapped on a graph $\mathcal{G}=(\mathcal{N}, \mathcal{E})$, where $\mathcal{N}$ represents the set of nodes (image locations) and $\mathcal{E}$ represents a neighboring system of the image grid (typically 4 or 8 in 2D). The labeling problem is then solved by minimizing:

$$
E(\mathbf{u})=\sum_{i \in \mathcal{N}} \Phi_{i}\left(u_{i}\right)+\alpha \sum_{(i, j) \in \mathcal{E}} \Psi_{i j}\left(u_{i}, u_{j}\right)
$$

where $u_{i} \in \mathcal{L}$ denotes the displacement label for location $i, \Phi_{i}($.$) is the unary$ potentials representing the data term and $\Psi_{i j}(.,$.$) are the pairwise potentials rep-$ resenting the interaction (smoothing) term. Due to the independence assumption of the unary data potentials $\Phi_{i}$, we require a point-wise similarity score. This constraint was relaxed in [9] by approximating a local score (e.g. mutual information) in neighborhoods defined by control points. There are few formulations of the traditional MRF for solving the discrete registration problem. When the smoothing term $\Psi_{i j}$ is a metric, the MRF energy can efficiently be optimized using graph cuts [10 8]. For more complex interaction terms, Glocker et al. 9] use a linear programming method (based on the primal-dual principle).

We make few modifications to the traditional MRF from Equation 3 to be able to map the registration problem to a random walker with priors [13. First, we relax the labeling system to continuous variables $u_{i}^{k}$ that represent the probability of node $i$ having the label $u^{k}$. Next, we consider a Gaussian MRF, where the interaction term has the form $\Psi_{i j}\left(u_{i}^{k}, u_{j}^{k}\right)=w_{i j}\left(u_{i}^{k}-u_{j}^{k}\right)^{2}$, with $w_{i j}$ being an image dependent edge weight (e.g. $w_{i j}=\exp \left(-\beta\left(J_{i}-J_{j}\right)^{2}\right)$ where $J_{i}$ represents the image intensity for location $i)$. Last, for defining the data term $\Phi_{i}\left(u_{i}^{k}\right)$, we consider a set of real-valued nodewise priors $\lambda_{i}^{k}$ that represents the probability density that the displacement vector at location $i$ has the value $\mathbf{d}^{k}$, $\lambda_{i}^{k}=\exp \left(-\gamma\left(I_{i}-J_{i+\mathbf{d}^{k}}\right)^{2}\right)$. By $J_{i+\mathbf{d}^{k}}$ we denoted the intensity of image $J$ at location $i$ displaced with $\mathbf{d}^{k}$.

With these three modifications, following 13 , we can define the registration energy corresponding to the label $u^{k}$ as the continuous-valued Gaussian MRF:

$$
E^{k}\left(u^{k}\right)=\sum_{i \in \mathcal{N}}\left(\sum_{l=1, l \neq k}^{K} \lambda_{i}^{l}\left(u_{i}^{k}\right)^{2}+\lambda_{i}^{k}\left(1-u_{i}^{k}\right)^{2}\right)+\alpha \sum_{(i, j) \in \mathcal{E}} w_{i j}\left(u_{i}^{k}-u_{j}^{k}\right)^{2}
$$

While space does not allow a rigorous interpretation of the above equation (details in [13]), intuitively, we see that when $\lambda_{i}^{k}$ is large (meaning that the displacement $\mathbf{d}^{k}$ matches the similarity score at location $i$ ) the data energy term encourages high probability values for label $u^{k}$ and small probability values for all other labels $u^{l}, l \neq k$. Compacting notations, we denote $L$, the combinatorial Laplacian matrix of the graph: 


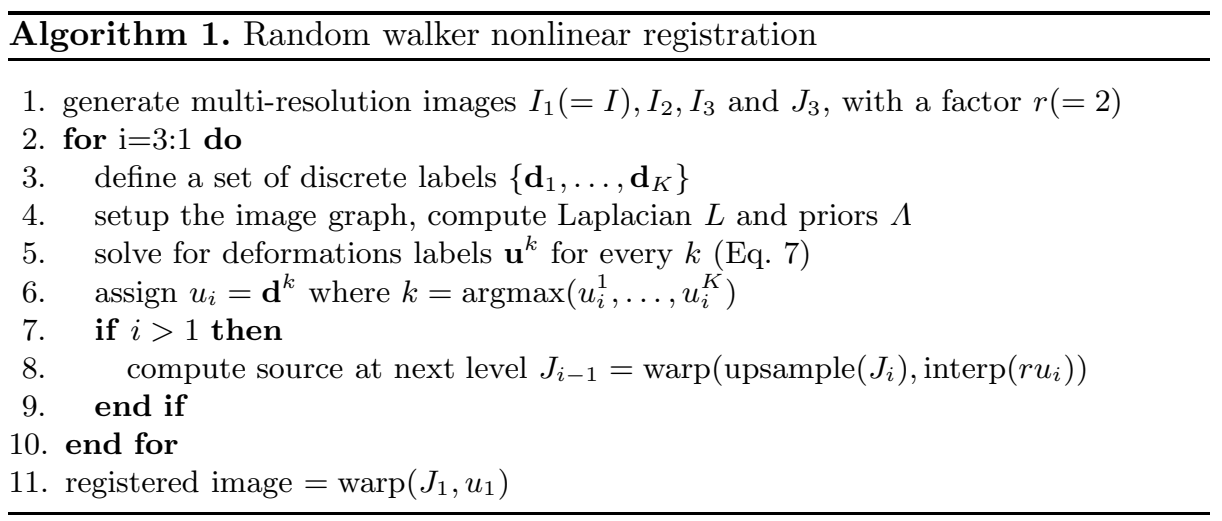

$$
L_{i j}= \begin{cases}d_{i}=\sum_{k} w_{i k} & \text { degree of } i \text { if } i=j \\ -w_{i j} & \text { if }(i, j) \in \mathcal{E} \\ 0 & \text { otherwise }\end{cases}
$$

and by $\Lambda^{k}=\operatorname{diag}\left(\lambda^{k}\right)$, the matrix having the values of $\lambda^{k}$ on the diagonal. Equation 4 can be written as:

$$
E^{k}\left(\mathbf{u}^{k}\right)=\sum_{l=1, l \neq k}^{K} \mathbf{u}^{k T} \Lambda^{l} \mathbf{u}^{k}+\left(1-\mathbf{u}^{k}\right)^{T} \Lambda^{k}\left(1-\mathbf{u}^{k}\right)+\alpha \mathbf{u}^{k T} L \mathbf{u}^{k}
$$

where $\mathbf{u}^{k}$ collects all nodes probabilities for label $k$ in a vector. The minimum of this energy is obtained when $\mathbf{u}^{k}$ is the solution of this equation:

$$
\left(\alpha L+\sum_{l=1}^{K} \Lambda^{l}\right) \mathbf{u}^{k}=\lambda^{k}
$$

which is a combinatorial Laplace equation of a graph augmented with a node for each label $u^{k}$ such that the weights on the new edges $(k, i)$ have value $\lambda_{i}^{k}$. The combined matrix on the left side of the equation is guaranteed to be positive definite and therefore the equation has a unique global solution that gives the nodes probabilities for the displacement labels $\mathbf{u}^{k}$.

\subsection{Multi-resolution Framework and Implementation Details}

The random walker algorithm is computationally expensive as well as memory expensive. The number of equation systems to be solved is the same as the number of displacement labels, and each of these equations has the number of variables equal to the number of pixels in the image. As the number of displacement labels can be quite large, especially in 3D, not only the solution of the linear systems is time-consuming, but also their assembly. We obtained an efficient approximation of the solution using a multi-resolution framework. Due to 


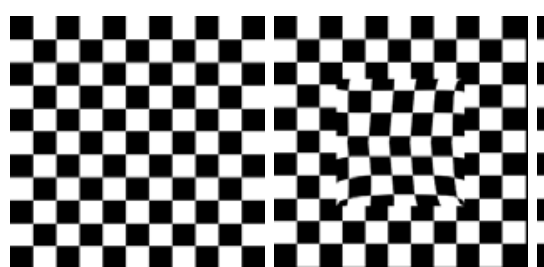

target

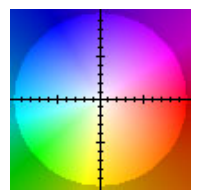

color coding deformed

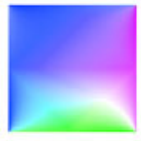

orig. def.

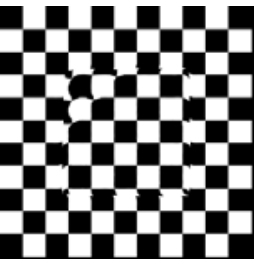

reg. with demon

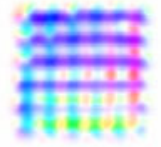

rec. def. demon

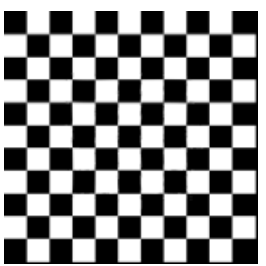

reg. with RW

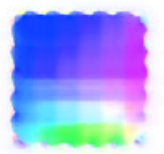

rec. def. RW

Fig. 1. Comparative results of recovered deformations for checkerboard image. From left to right, the top row shows the target image, the deformed image and the SSD error of the registered images with demon's and RW methods. Bottom row shows the angle and magnitude color coding convention, the ground truth deformations and the recovered deformations using demon's and RW methods.

this multiresolution approach, even though the RW solution is optimal at each resolution level, the composite solution is no longer guaranteed to be optimal.

The multi-resolution images were obtained by downsampling the original images based on nearest neighbor interpolation. We defined deformations in an incremental way propagating deformations obtained at a lower resolution to the next higher resolution level. This approximation is carried out by an interpolation based on Delaunay triangulation after scaling the low resolution field by the multi-resolution scale factor. At each resolution we compute the remaining deformations by solving the sparse linear equation system 7 for each discrete label (in practice only $K-1$ times as we impose sum of unity for the probabilities $\left.\sum_{k} u_{i}^{k}=1\right)$. At each level the magnitude and number of expected deformations encoded as discrete labels $\left\{\mathbf{d}^{1}, \mathbf{d}^{2}, \ldots, \mathbf{d}^{K}\right\}$ decreases. The final displacements $u_{i}$ are taken as the ones with maximum probability among all labels $u_{i}=\mathbf{d}^{k}$ where $k=\operatorname{argmax}\left(u_{i}^{1}, \ldots, u_{i}^{K}\right)$. The algorithm is summarized in Algorithm 1 .

\section{Experiments}

We present results of our experiments on real and synthetic data. We compared the performance of the proposed RW registration with a traditional demon's implementation 14. The set of parameters are optimized to achieve best SSD scores (ex. $\alpha=1$, Gaussian weights $w_{i j}$ with $\beta=0.005$, priors $\lambda_{i}^{k}$ with $\gamma=10^{-5}$ for real data). For all experiments we used 3 levels of resolution generated by a scale factor of 0.5 and 0.25 . The number of displacement labels at each level 
Table 1. Comparative numerical results for the three datasets

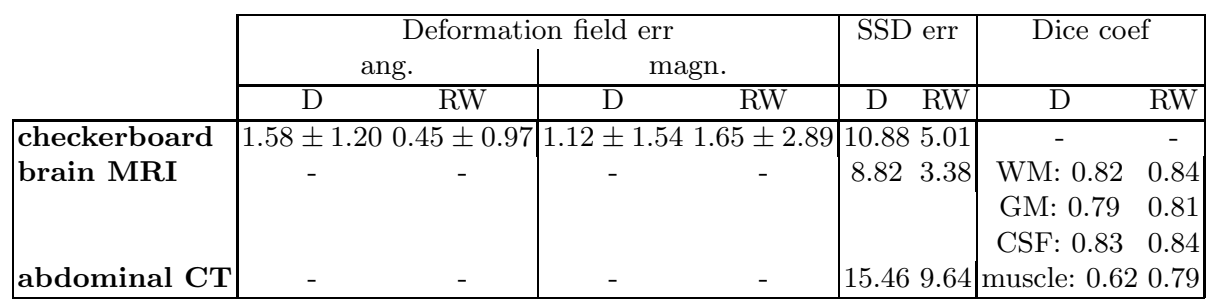

is dependent on the initial image size (ex. low-high resolution : 60,40,30 for an initial $256 \times 256$ image, corresponding to a displacement range of about [ $-15,15]$, $[-10,10],[-7,7]$ pixels, respectively). The experiments were run on MATLAB using Intel Core 2 Duo Processor of $2.10 \mathrm{GHz}$ with 4 GB RAM. The algorithm took about $200 \mathrm{sec}$. to complete on a $256 \times 256$ image. Most time was taken by MATLAB's sparse linear equation solver.

\subsection{Quality of Recovered Deformations}

For testing the accuracy of the recovered deformation field we synthetically deformed a checkerboard image with a known deformation field. We tested how deformations are recovered by the RW registration method and the demon's algorithm. We measured the angular and magnitude errors of the recovered deformation fields as well as the SSD error between target and registered images. Qualitative results are presented in Figure 1 and numerical scores in Table 1. We color coded deformations using the same convention as for optic flow as shown in bottom-left of Figure 1. We notice that the recovered deformation fields using RW registration has less artifacts, and the recovered deformations are closer in orientation to the original ones. The magnitude of recovered deformations using RW is slightly larger than the ones recovered using demons, probably due to the fact that regularization is imposed at the energy level for which we obtain a global solution as opposed to demon's iterative approach.

\subsection{Results on Real Medical Data}

For the experiments with real data, we again compared our RW method and demon's algorithm. To quantify results we measured SSD error between registered images and the target image. Also, both datasets had some ground truth segmentations (WM/GM/CSF for brain MRI data and muscle in the abdominal CT data). We calculated the dice coefficients between ground truth segmentations in the target image, and segmentations from the source image warped on the space of the target image using the recovered deformation field. Note that a larger, closer to 1 value for a dice coefficient indicates a better segmentation.

The first experiment uses the brain MRI dataset from Internet Brain Segmentation Repository 1. We performed registration between two patients, both

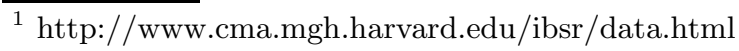



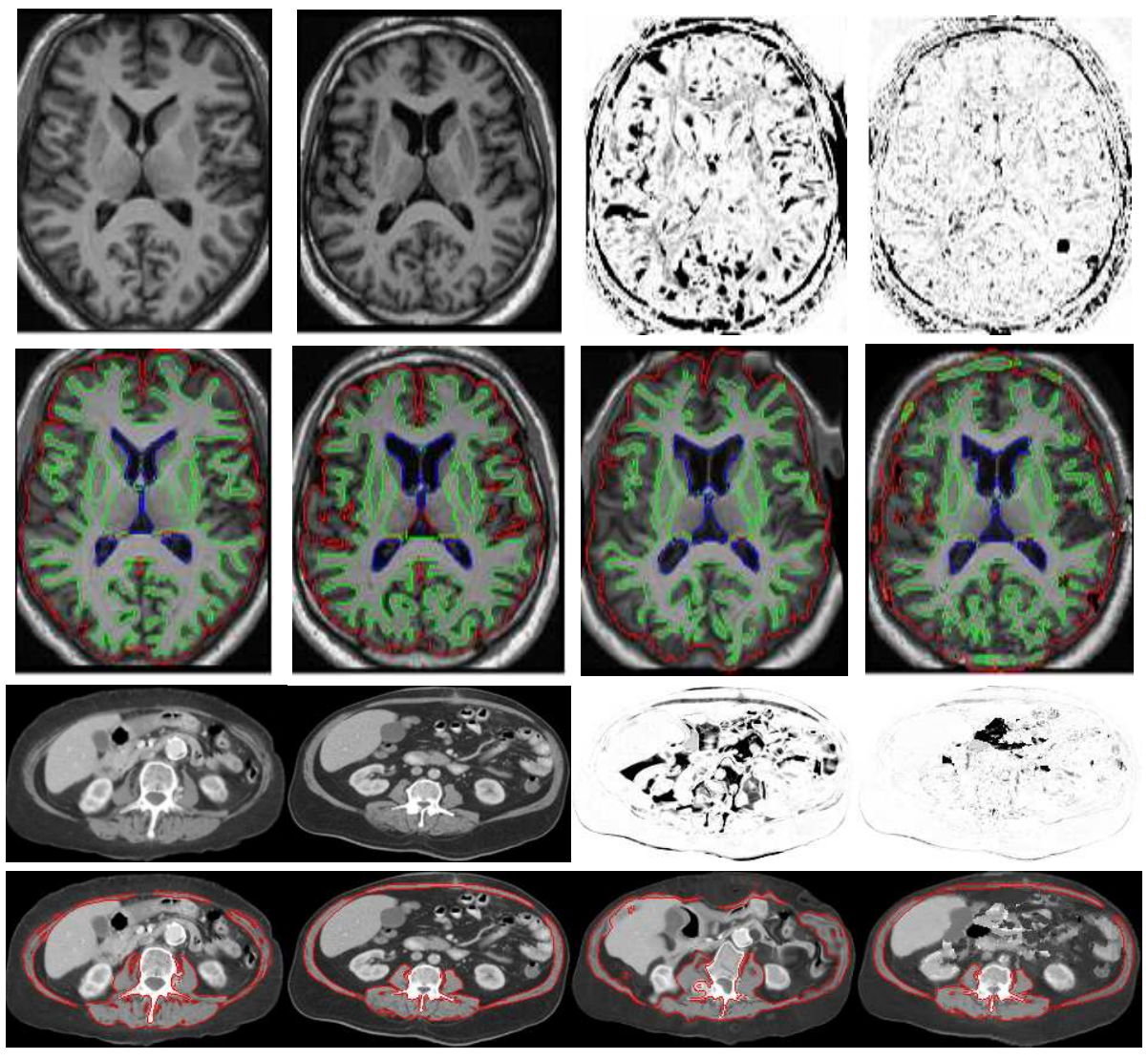

source

target

reg. demons

reg. RW

Fig. 2. Results for real data. Left to right: (row 1) brain MRI data - source, target, SSD error between target and registered image (bright for small, dark for large errors); (row 2) segmentations on source and target images,warped segmentations on registered images; (row 3,4) same for abdominal CT data.

from the database. The dataset contains WM,GM and CSF labels. As shown in the first two rows of Figure 2 and in Table 1, the RW method has better performance both visually and quantified compared to the demon's algorithm.

In a second experiment we registered two abdominal CT images from a local cancer institute. Notice the large difference between the two datasets. Demon's method was not able to recover the large deformations but the RW's registration that recovers a global minimum was much better. As the muscle exist in both images, and is therefore the part that is expected to match, we measured the dice coefficient on the muscle segmentation. Ground truth segmentation was provided by a medical student. Figure 2 and Table 1 present the results. 


\section{Discussion}

We have presented a discrete method for non-rigid image registration based on the random walker method. The new formulation has several advantages: at each resolution level, we globally minimize a convex energy, with a regularization term that is image dependent thus being able to accommodate different elasticity depending on the tissue type.

As future work, we are looking into a more efficient implementation of our method. One option is to use a lower-dimensional deformation model like the FFD model that computes displacements only at control points. This approach would also allow approximating non-local data potentials (e.g. mutual information) in neighborhoods around the control points (similar to 9]). This technically violates the independence assumption, but practically the loss of optimality at a particular resolution may be compensated by the richer non-local measure.

\section{References}

1. Zikic, D., Kamen, A., Navab, N.: Unifying characterization of deformable registration methods based on the inherent parametrization. In: Fischer, B., Dawant, B.M., Lorenz, C. (eds.) WBIR 2010. LNCS, vol. 6204, pp. 161-172. Springer, Heidelberg (2010)

2. Huang, X., Metaxas, D.T.: Metamorphs: Deformable shape and texture models. In: CVPR (2004)

3. Pennec, X., Cachier, P., Ayache, N.: Understanding the "demon's algorithm": 3d non-rigid registration by gradient descent. In: Taylor, C., Colchester, A. (eds.) MICCAI 1999. LNCS, vol. 1679, pp. 597-605. Springer, Heidelberg (1999)

4. Vercauteren, T., Pennec, X., Malis, E., Perchant, A., Ayache, N.: Insight into efficient image registration techniques and the demons algorithm. In: Karssemeijer, N., Lelieveldt, B. (eds.) IPMI 2007. LNCS, vol. 4584, pp. 495-506. Springer, Heidelberg (2007)

5. Thirion, J.P.: Image matching as a diffusion process: an analogy with maxwell's demons. Medical Image Analysis 2(3), 243-260 (1998)

6. Zikic, D., Baust, M., Kamen, A., Navab, N.: Generalization of deformable registration in riemannian sobolev spaces. In: Jiang, T., Navab, N., Pluim, J.P.W., Viergever, M.A. (eds.) MICCAI 2010. LNCS, vol. MICCAI, pp. 586-593. Springer, Heidelberg (2010)

7. Stefanescu, R., Pennec, X., Ayache, N.: Grid powered nonlinear image registration with locally adaptive regularization. In: Medical Image Analysis: MICCAI 2003 Special Issue, vol. 8(3), pp. 325-342 (2004)

8. Tang, L., Hamarneh, G., Abugharbieh, R.: Reliability-driven, spatially-adaptive regularization for deformable registration. In: Fischer, B., Dawant, B.M., Lorenz, C. (eds.) WBIR 2010. LNCS, vol. 6204, pp. 173-185. Springer, Heidelberg (2010)

9. Glocker, B., Komodakis, N., Tziritas, G., Navab, N., Paragios, N.: Dense image registration through mrfs and efficient linear programming. Medical Image Analysis $12(6), 731-741(2008)$

10. Tang, T.W., Chung, A.C.: Non-rigid image registration using graph-cuts. In: Ayache, N., Ourselin, S., Maeder, A. (eds.) MICCAI 2007, Part I. LNCS, vol. 4791, pp. 916-924. Springer, Heidelberg (2007)

11. Boykov, Y., Veksler, O., Zabih, R.: Fast approximate energy minimization via graph cuts. IEEE Trans. PAMI 23(11), 1222-1239 (2001) 
12. Grady, L.: Random walks for image segmentation. IEEE Trans. PAMI 28(11), 1768-1783 (2006)

13. Grady, L.: Multilabel random walker image segmentation using prior models. In: CVPR, pp. 763-770 (2005)

14. Kroon, D.J.: Deamon's implementation, http://www . mathworks. com/matlabcentral/fileexchange/ 